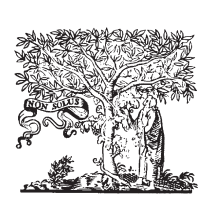

\title{
Hirschsprung's disease
}

\author{
AQ: 1 Simon E. Kenny, ${ }^{a}$ Paul K. H. Tam, ${ }^{b}$ Mercè Garcia-Barcelo ${ }^{b}$ \\ From the ${ }^{a}$ Department of Paediatric Surgery, Alder Hey Children's NHS Foundation Trust and Division of Reproductive \\ and Developmental Medicine, University of Liverpool, Liverpool, UK; and \\ ${ }^{b}$ Department of Surgery, Li Ka Shing Faculty of Medicine, The University of Hong Kong, Hong Kong, P. R. China.
}

\section{KEYWORDS}

Hirschprungs disease;

Aganglionosis;

Etiology;

Genetics

\begin{abstract}
Hirschsprung's disease (HSCR) is characterized by absence of the enteric nervous system in a variable portion of the distal gut. Affected infants usually present in the days after birth with bowel obstruction. Despite surgical advances, long-term outcomes remain variable. In the last 2 decades, great advances have been made in understanding the genes and molecular biological mechanisms that underlie the disease. In addition, our understanding of normal enteric nervous system development and how motility develops in the developing fetus and infant has also increased. This review aims to draw these strands together to explain the developmental and biological basis of HSCR, and how this knowledge may be used in future to aid children with HSCR.

(C) 2010 Published by Elsevier Inc.
\end{abstract}

Hirschsprung's disease (HSCR) is the commonest congenital gut motility disorder and is characterized by a lack of ganglion cells (aganglionosis) in a variable length of distal gut. Affected infants usually present shortly after birth with signs of distal intestinal obstruction that are invariably fatal if left untreated. Current definitive treatment involves surgery to resect the aganglionic bowel segment and "pullthrough" and anastomosis of normally innervated (ganglionic) gut close to the anal margin. Although broadly successful in the majority of patients, challenges are encountered in the management of children with more extensive aganglionosis and those who experience repeated bouts of enterocolitis. ${ }^{1}$ Furthermore, in the long term up to $75 \%$ of children will have some form of continence or constipation problem, and $10 \%$ have symptoms sufficiently severe to warrant a permanent colostomy. Clearly, an understanding of the biological and developmental basis of aganglionosis is extremely relevant in understanding the reasons for such variability in biological presentation and also in formulating novel treatments

\footnotetext{
Address reprint requests and correspondence: Mr Simon E. Kenny, Department of Paediatric Surgery Alder Hey Children's NHS Foundation Trust Eaton Rd, Liverpool L12 2AP, UK.

E-mail address: simon.kenny@liv.ac.uk.
}

for children with HSCR in future. It has long been noted that HSCR can be familial and also associated with a range of syndrome conditions. This review will therefore address the underlying developmental and biological basis of HSCR with particular emphasis on its' genetic basis.

\section{Incidence and associated anomalies}

Demographic studies have shown a remarkably constant incidence of HSCR of approximately 1 in 5000 in both hemispheres, although most epidemiologic studies have been confined to the Caucasian Diaspora, and thus there may be as yet undefined interracial differences. Evidence for this comes from a Californian survey in which the authors found significant interracial differences in incidence of HSCR: 1:10,000 births in Hispanic subjects, 1:6667 in white subjects, 1:4761 in black subjects, 1:3571 in Asian subjects. ${ }^{2}$ Differing levels of consanguinity in different populations may explain some of the differences, but the authors of recent genetic studies concerning frequencies of HSCR-associated mutations point to different frequencies in different ethnic populations. ${ }^{3}$ 
Table 1 Additional anomalies in Hirschsprung's disease

\begin{tabular}{|c|c|}
\hline Anomaly & Example \\
\hline \multicolumn{2}{|l|}{$\begin{array}{l}\text { Neural crest-related } \\
\text { anomalies }\end{array}$} \\
\hline Sensorineural deafness & $\begin{array}{l}\text { Congenital central } \\
\text { hypoventilation syndrome } \\
\text { Isolated sensorineural } \\
\text { deafness } \\
\text { Waardenburg syndrome }\end{array}$ \\
\hline Cardiovascular skeletal & DiGeorge syndrome \\
\hline & $\begin{array}{l}\text { Postaxial polydactyly and } \\
\text { heart defect } \\
\text { CRASH syndrome (X-linked } \\
\text { aqueductal stenosis) } \\
\text { Congenital muscular dystrophy }\end{array}$ \\
\hline Cleft palate & $\begin{array}{l}\text { Goldberg Sphrintzen syndrome } \\
\text { DiGeorge syndrome }\end{array}$ \\
\hline Systemic anomalies & $\begin{array}{l}\text { Neurofibromatosis type } 1 \\
\text { Multiple endocrine neoplasia } \\
\text { type } 2 \mathrm{~A} \\
\text { Multiple endocrine neoplasia } \\
\text { type } 2 \mathrm{~B} \\
\text { Smith-Lemli Opitz syndrome } \\
\text { Dysautonomias }\end{array}$ \\
\hline $\begin{array}{l}\text { Other anomalies } \\
\text { Trisomy } 21 \\
\text { Microcephaly } \\
\text { Mental retardation } \\
\text { Inguinal hernia } \\
\text { Small bowel atresia } \\
\text { Duodenal atresia } \\
\text { Genital reproductive tract } \\
\text { Undescended testes }\end{array}$ & \\
\hline $\begin{array}{l}\text { Regional anomalies } \\
\text { Rectal stenosis } \\
\text { Anal stenosis } \\
\text { Imperforate anus } \\
\text { Colonic atresia }\end{array}$ & \\
\hline
\end{tabular}

In the most common, "classical" form of HSCR, aganglionosis is restricted to the rectosigmoid region and is referred to as "short segment" disease. This variant accounts for more than $80 \%$ of cases. In the remaining cases, colonic aganglionosis is more extensive and may involve distal small intestine. Total enteric aganglionosis is both rare and associated with high morbidity and mortality. There is a strong male gender bias, with male patients being affected 2 to 4 times more commonly than female ones, although this bias is lost in children with more extensive aganglionosis.

Important clues as to which genes are involved in HSCR have come from the study of the pattern of associated T1 malformations that occur in $4 \%$ to $35 \%$ of cases (Table 1). Knowledge of associated anomalies is also important in the course of genetic counseling and because of potentially deleterious known associations-medullary thyroid carcinoma as part of multiple endocrine neoplasia syndrome Type 2 B (MEN2B) is perhaps the best example. One of the commonest associated malformations is Down's syndrome (trisomy 21), which carries a 100-fold greater risk for HSCR than the normal population. ${ }^{4}$

As discussed in this article, the enteric nervous system is of neural crest origin, and hence HSCR is regarded as a neurocristopathy. Therefore, it is unsurprising that it is associated with other neurocristopathies because factors affecting the migration of enteric neuroblasts may well affect the migration, differentiation, or survival of other neural crest derived cells, for example, Shah-Waardenburg (WS4).

\section{The role of the enteric nervous system in determining gut motility}

Gut motility is a complex process mediated by interaction between intestinal smooth muscle (SM), "pacemaker" cells (interstitial cells of Cajal; ICC), and the enteric nervous system (ENS). Unlike in the heart, intestinal SM cells are unable to generate rhythmic electrical slow waves. In the last 2 decades it has been established that ICC are responsible for slow-wave activity in muscle that can propagate to adjacent muscle. ${ }^{5,6}$ Although ICC-generated slow waves result in some contractile activity and a tendency for intestinal contents to be propagated in a craniocaudal direction, the ENS is essential for more widespread coordination plus modulation of amplitude and frequency of SM contraction to generate the 2 main types of contractions in the gut: segmentation and peristaltic waves. Both occur in the absence of extrinsic innervation but require an intact myenteric plexus. Colonic motility is quite distinct from small intestinal motility, and regionalization of contractions in different regions of the colon occurs. ICC-mediated slow-wave activity causes colonic contractions when the depolarization is of sufficient amplitude. At the end of the gastrointestinal tract sits the internal sphincter, a specialized thickening of circular SM within the distal rectum. It maintains a state of tonic contraction thus maintaining continence in association with the external sphincter. Distension of the rectum, typically with feces, results in an ENS-dependent reflexive relaxation of the sphincter (rectoanal inhibitory reflex). To achieve these functions, the ENS is extensive and contains a diverse range of neuronal phenotypes characterized by neurotransmitters and morphology; see Hao and Young for review. ${ }^{7}$ The critical role of the ENS is illustrated by the obstruction that occurs in children with HSCR (in which there is congenital absence of the distal ENS): colonic mass movements are unable to propagate through the aganglionic segment that remains in a tonic state. Furthermore, the presence of feces in the rectum fails to elicit relaxation in the aganglionic anal sphincter, which contributes to the obstructive picture seen clinically.$$
1
$$

$$
4
$$$$
\text { , }
$$

$$
8
$$$$
\text { 列 }
$$

$$
2
$$$$
4
$$$$
5
$$

$$
7
$$$$
\text { . }
$$

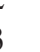

$$
4
$$$$
7
$$

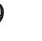

$$
\text { 这 }
$$




\section{Onset of gut motility in the fetus, normal, and premature neonates}

There is a remarkable paucity of data on the ontogeny of human gut motility that reflects the inherent difficulties in studying the developing human. By late gestational age, fetal swallowing results in ingestion of amniotic fluid that is propagated through the gut. ${ }^{8}$ Painstaking antenatal ultrasonographic studies of fetal gut motility demonstrate fetal gastric emptying occurring at 24 weeks and assuming more mature patterns by term. ${ }^{9}$ Small intestinal peristalsis is rarely observed before 29 weeks and subjective observation suggests active waves of small intestinal peristalsis are infrequently seen before 35 weeks of gestation. ${ }^{10}$ Similarly, ultrasound studies on human fetal internal sphincter development suggest that rhythmic contractions commence in the third trimester. ${ }^{11}$ Preterm infants appear to manifest similar patterns of onset of gastrointestinal motility, exhibiting markedly delayed gastrointestinal transit times when compared with adults. In the small intestine of preterm children, disorganized peristalsis is seen before the third trimester, with migrating motor complexes being observed in human small intestine after 33 weeks of gestation. ${ }^{12}$

There is a marked lack of data on colonic motility in human preterms. Some evidence can be gleaned from animal studies in that, in common with humans, intestinal contents are propagated through the bowel before birth. The authors of recent studies suggest that effective colonic contractions do occur but that these are not mediated by the ENS. ${ }^{13,14}$ Taken together, in both animals and humans although the main components regulating gut motility are present by 14 weeks of gestation, it seems likely that the ENS is relatively quiescent until late in gestation and gut motility is controlled by other factors, such as ICC. This explains our failure to detect HSCR antenatally as if the colonic ENS is not functional until birth no bowel dilatation will be detected on ultrasound. This is also seen clinically, in that invariably the abdominal distension is progressive after birth rather than being clinically detectable at the moment of birth.

\section{Neural crest origin of the enteric nervous system and the pathogenesis of Hirschsprung's disease}

Neural crest ablation studies and chick-quail chimaera experiments have shown that ENS neurons and glia are derived from the vagal segment of the neural crest. ${ }^{15,16} \mathrm{Va}$ gally derived neural crest cells (NCCs) migrate along the course of the vagus nerves, enter the foregut mesenchyme, and spread in a craniocaudal direction throughout the gastrointestinal tract. In humans the process takes 7 weeks, with neural crest derivatives entering the foregut at 5 weeks, reaching the distal ileum by 7 weeks, the midcolon by 8 weeks, but taking a further 4 weeks to reach the distal rectum. ${ }^{17,18}$ This slowing in rate of colonization of the distal 106 gut is caused by growth of the bowel rather than a reduction in velocity of migration. In mammals, there is an additional sacral contribution to the colonic ENS but migration of sacral crest cells follows vagal neural crest colonization and these cells in isolation are insufficient in isolation to rescue the HSCR phenotype. ${ }^{19}$

The vagal sourced NCCs in the distal rectum migrate further than any other cells during embryogenesis. It is therefore not surprising that factors affecting proliferation, survival, migration, or differentiation of NCCs results in aganglionosis of the distal gut. Although important advances have been made in identifying the complex genetic picture in HSCR, unraveling the biological mechanisms that prevail in normal neural crest colonization of the gut, and how this goes wrong in HSCR has been a formidable technical challenge because of the inaccessibility of the developing bowel and the lack of reliable in vivo markers.

Mathematical modeling coupled with experimental manipulation of chick-quail chimaeras of gut explants suggest that cell proliferation at the vanguard of migrating NCC drives colonization of aganglionic gut with HSCR, resulting from discordance between the rate of cell proliferation and elongation by growth of the developing gut. ${ }^{20,21}$ In recent years, the use of the green fluorescent protein gene as a reporter of NCC expression in explanted mammalian and avian embryonic gut cultures, or in translucent zebrafish, in combination with time-lapse photography has allowed the pattern of neural crest migration and ENS formation to be better understood. Furthermore, experimental manipulation of the embryonic gut environment and gene expression has allowed insights into the pathogenesis of aganglionosis. What has emerged is a complex spatiotemporal interaction between migrating cells, developing neurons, and the gut.

Assumptions about the actions of genes made from isolated neural crest cells in vitro have been found wanting as cells respond to the same cues differently according to their location in the gut and gestational age. Chains of immature neuroblasts migrate through the developing gut and leave a scaffold that subsequent cells follow. This migration has been shown to be directionally driven by noncanonical Wnt signaling, causing contact inhibition; ${ }^{22}$ although unpredictable at a single cell level, this seems stereotyped at the organ level. In particular, a single chain of cells appear to extend along the mesenteric border of the cecum well in advance of the rest of the advancing wave of colonization. ${ }^{23}$ There is some evidence that migrating cells may be routed along the developing vasculature. ${ }^{24}$ Migrating cells have been shown to undergo cell division to increase cell numbers. Furthermore, apoptotic control mechanisms may control the final neuronal density in the gut because inhibition of apoptosis during NCC colonization results in hyperganglionosis. ${ }^{25}$ Only a small proportion of migrating cells express neuronal markers and these migrate more slowly. ${ }^{26}$ Increasing cell maturation as reflected by expression of neuronal or glial phenotype and subsequent neurotransmitter expression is 
associated with loss of migratory ability. ${ }^{26}$ Microenvironmental factors in the noninnervated colon, such as overexpression of laminin, have been suspected to be implicated in the pathogenesis of $\mathrm{HSCR}^{27,28}$ and documented in the aganglionic colon of children with HSCR. ${ }^{29}$ In support of these observations, recent gut explant experiments point to agedependent changes in the gut resulting in restriction of NCC migration into older bowel. ${ }^{30,31}$ Clearly in HSCR, several genetic and environmental factors interact to result in failure of colonization of the distal intestine (see the section 2 "Hirschsprung's disease and genes").

A variety of experiments in which small numbers of neural crest-derived cells were cultured in aganglionic bowel has demonstrated that relatively small numbers of these cells can engage in extensive colonization and formation of both neurons and glia expressing a range of phenotypic markers and expressing appropriate neurotransmitters. ${ }^{32,33}$ Such observations point to the existence of a reservoir of "stem-cells" within the migrating wave: cells with extensive proliferative and differentiative capacity. As will be discussed later, the existence of these cells may point to a future stem-cell based therapy for HSCR. It should be remembered that at the same time as NCC are migrating and colonizing the gut the gut is maturing in many ways that will impact on future motility. SM and ICC are differentiating from mesenchyme and later in gestation functional connections are forming between neurones, ICC, and SM (for review, consult Burns et $\mathrm{al}^{32}$ ).

\section{Hirschsprung's disease and genes}

HSCR is a complex genetic disease with a low, sex-dependent penetrance (frequency of mutation carriers who have HSCR) and variability in the length of the aganglionic segment (for review, see Tam and Garcia-Barcelo ${ }^{33}$ and 3 Arnold et $\mathrm{al}^{36}$ ). The genetic diversity observed in HSCR can be attributed to the cascade of molecular and cellular events that take place during the ENS development as outlined above. Disruption of coding sequences resulting in functional changes to gene products of any of the genes responsible for neural crest cell migration, proliferation, differentiation, survival or that alter the permissive environment for NCC migration holds the potential for failure of ENS deT2 velopment resulting in HSCR (Table 2). The HSCR phenotype may result from mutations in single or multiple genes. The existence of individuals with major HSCR-causing mutations who do not manifest the disease underlines the complex multigenic mechanisms of ENS formation and also potentially the role played by environmental factors.

Furthermore, the existence of an overrepresentation of mutations and/or SNPs in gene-receptor complexes, such as ret-GDNF and/or 3rd edn/ENDRB, ${ }^{34,35}$ when compared with controls suggests subtle influences of both major genereceptor complexes in determining HSCR susceptibility. The role of identified genes in shaping the demographic presentation of HSCR is also beginning to be understood. For example, an association between RET and chromosome 21 gene dosage has recently been described. ${ }^{36}$ The male preponderance observed in isolated short-segment HSCR may potentially be explained by the recent finding of reduced levels of ECE-1 and endothelin-3 mRNA in normal male mouse bowel versus females. ${ }^{37}$ In the same report, comparison of male versus female cultured explanted bowel from heterozygous mice with a RetDN mutation (showing reduced but not absent Ret activity) showed reduced colonization in male mice. Supplementation of male cultured bowel with endothelin-3 peptide resulted in a significant increase in the rate of bowel colonization. Thus, there is increasing evidence that sex-related differences in endothelin-3 expression, on a background of genetic susceptibility, may account for the male overrepresentation in HSCR.

\section{Modifying genes and interaction between signaling pathways}

As indicated previously, the successful colonization of the gut by the ENS precursors depends on the network of interacting molecules. Conceivably, there should be a functional and genetic link among these molecules for them to interact. Interaction between pathways requires not only coordination among the pathway members but also with those molecules that mediate their interaction. There is increasing evidence of interactions between genes in apparently different signaling pathways. ${ }^{33}$

\section{Hirschsprung's disease and stem cells}

The discovery of a subset of cells with significant proliferative and differentiative capacity within the migrating wave of NCCs has given rise to the hope that these cells could potentially represent enteric nervous system stem cells (ENSC). Stem cells are characterized by their capacity for asymmetric cell division, both self-renewing and producing daughter cells that have the ability to proliferate and form a range of cell types. Putative ENSCs should therefore be demonstrably immortal, clonal, and capable of proliferating to form neurons and glia. That these properties have been demonstrated in mouse NCC strongly supports the existence of ENSCs. ${ }^{38-43}$ More recently, human ENSCs have been isolated from children and adults with and without HSCR that can be numerically expanded in vitro and transplanted into animal models of HSCR where they have proliferated and formed neurons and glia. $^{38,44,45}$ Furthermore, neuronal function has been demonstrated. ${ }^{14}$

These results appear promising for future clinical applications. Nevertheless significant obstacles remain. The behavior of human ENSCs in the more mature environment of the neonatal gut needs to be assessed and a robust reproducible and safe form of ENSC transplantation into the right 
\begin{tabular}{|l|l|l|l|l|l|}
\hline tapraid2/zp5-spsu/zp5-spsu/zp599909/zp52118d09z & mandgaonkarg & S=1 & 4/28/10 & Art: 50313 & ce: 22 \\
\hline
\end{tabular}

Kenny, Tam, and Garcia-Barcelo Hirschsprung's Disease

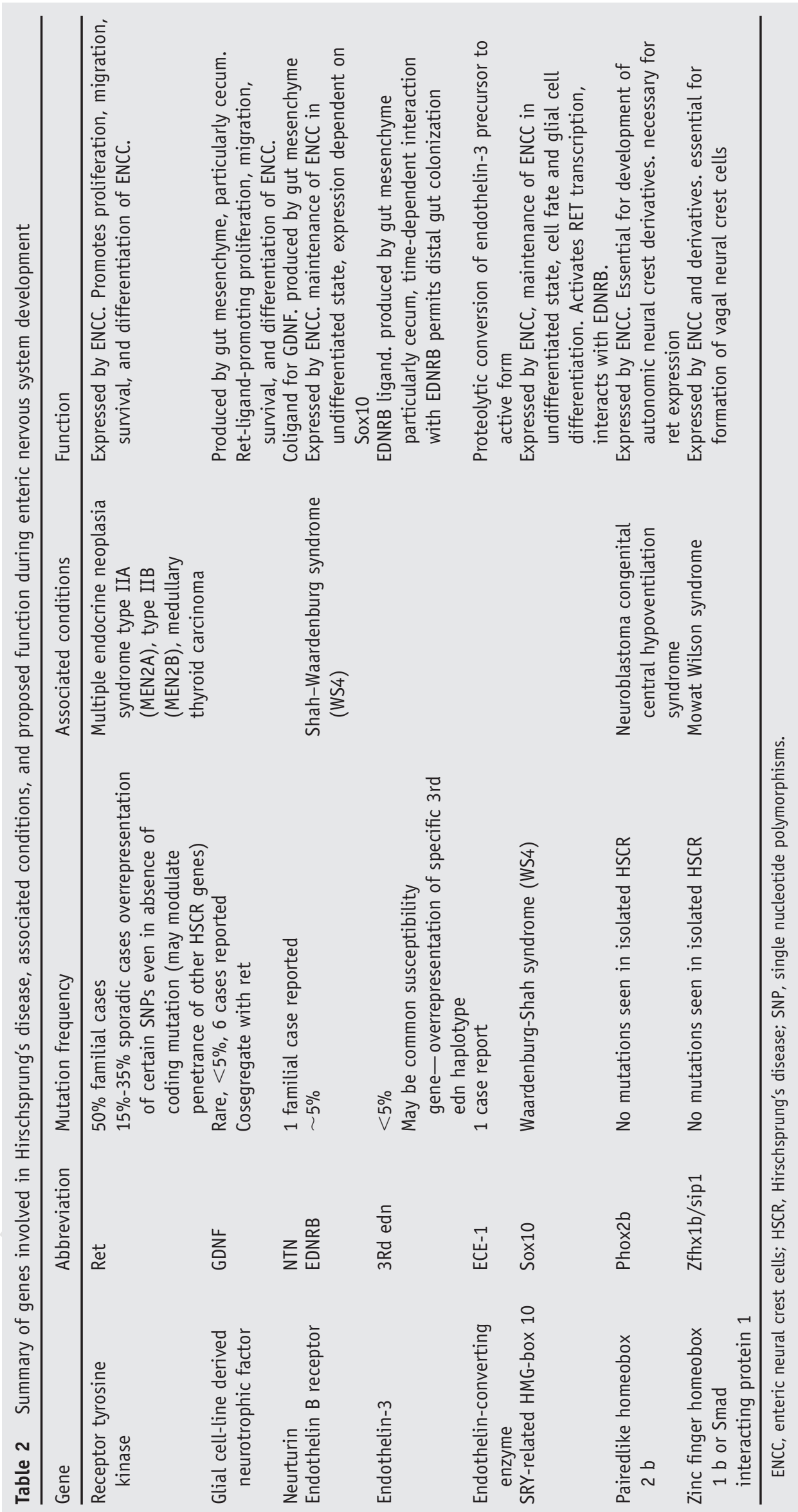


environment developed. Furthermore, long-term studies are necessary to demonstrate the genomic stability of transplanted cells to assess potential tumor risk. In addition, techniques that permit in vivo tracking of transplanted ENSC by the use of such technologies as green fluorescent protein labeling or stable integration of nanoparticles, such as superparamagnetic iron oxide nanoparticles within animal models is essential to understand the potential of ENSCs to migrate beyond the bowel.

In conclusion, the last 2 decades have yielded huge advances in our understanding of the developmental and biological basis of Hirschsprung's disease and gut motility in general. Significant challenges remain but increasing understanding of this subject may lead to prediction of HSCR risk and potentially to new treatments and improved outcomes for this condition.

\section{Acknowledgments}

The authors would like to acknowledge the research grants HKU 765407M and HKU 775907M from the Hong Kong Research Grants Council to M.G.B. and P.T., respectively. S.K. is supported by grants from Action Medical Research, Medical Research Council, and the Children's Research Fund.

\section{References}

1. Baillie CT, Kenny SE, Rintala RJ, et al: Long-term outcome and colonic motility after the Duhamel procedure for Hirschsprung's disease. J Pediatr Surg 34:325-329, 1999

2. Torfs C: An epidemiological study of Hirschsprung's disease in a multiracial Californian population. Presented at the The Third International Meeting: Hirschsprung disease and related neurocristopathies, Evian, 1998

3. Emison ES, McCallion AS, Kashuk CS, et al: A common sex-dependent mutation in a RET enhancer underlies Hirschsprung disease risk. Nature 434:857-863, 2005

4. Ikeda K, Goto S: Additional anomalies in Hirschsprung's disease: An analysis based on the nationwide survey in Japan. Z Kinderchir 41: 279-281, 1986

5. Sanders KM: Interstitial cells of Cajal at the clinical and scientific interface. J Physiol 576:683-687, 2006

6. Ward SM, Sanders KM, Hirst GD: Role of interstitial cells of Cajal in neural control of gastrointestinal smooth muscles. Neurogastroenterol Motil 16:112-117, 2004 (suppl 1)

7. Hao MM, Young HM: Development of enteric neuron diversity. J Cell Mol Med 13:1193-1210, 2009

8. McLain C: Amniography studies of the gastrointestinal motility of the human fetus. Am J Obstet Gynecol 86:1079-1087, 1963

9. Sase M, Miwa I, Sumie M, et al: Gastric emptying cycles in the human fetus. Am J Obstet Gynecol 193:1000-1004, 2005

10. Goldstein I, Lockwood C, Hobbins JC: Ultrasound assessment of fetal intestinal development in the evaluation of gestational age. Obstet Gynecol 70:682-686, 1987

11. Bourdelat D, Muller F, Droulle P, et al: Anatomical and sonographical studies on the development of fecal continence and sphincter development in human fetuses. Eur J Pediatr Surg 11:124-130, 2001
12. Berseth CL: Gastrointestinal motility in the neonate. Clin Perinatol 23:179-190, 1996

13. Anderson RB, Enomoto H, Bornstein JC, et al: The enteric nervous system is not essential for the propulsion of gut contents in fetal mice. Gut 53:1546-1547, 2004

14. Lindley RM, Hawcutt DB, Connell MG, et al: Human and mouse enteric nervous system neurosphere transplants regulate the function of aganglionic embryonic distal colon. Gastroenterology 135:205-216: e206, 2008

15. Le Douarin NM, Teillet MA: The migration of neural crest cells to the wall of the digestive tract in avian embryo. J Embryol Exp Morphol 30:31-48, 1973

16. Yntema CL, Hammond WS: The origin of intrinsic ganglia of trunk viscera from vagal neural crest in the chick embryo. J Comp Neurol 101:515-541, 1954

17. Fu M, Tam PK, Sham MH, et al: Embryonic development of the ganglion plexuses and the concentric layer structure of human gut: A topographical study. Anat Embryol (Berl) 208:33-41, 2004

18. Wallace AS, Burns AJ: Development of the enteric nervous system, smooth muscle and interstitial cells of Cajal in the human gastrointestinal tract. Cell Tissue Res 319:367-382, 2005

19. Burns AJ, Douarin NM: The sacral neural crest contributes neurons and glia to the post-umbilical gut: Spatiotemporal analysis of the development of the enteric nervous system. Development 125:43354347, 1998

20. Landman KA, Simpson MJ, Newgreen DF: Mathematical and experimental insights into the development of the enteric nervous system and Hirschsprung's disease. Dev Growth Differ 49:277-286, 2007

21. Simpson MJ, Zhang DC, Mariani M, et al: Cell proliferation drives neural crest cell invasion of the intestine. Dev Biol 302:553-568, 2007

22. Carmona-Fontaine C, Matthews HK, Kuriyama S, et al: Contact inhibition of locomotion in vivo controls neural crest directional migration. Nature 456:957-961, 2008

23. Young HM, Bergner AJ, Anderson RB, et al: Dynamics of neural crest-derived cell migration in the embryonic mouse gut. Dev Biol 270:455-473, 2004

24. Nagy N, Mwizerwa O, Yaniv K, et al: Endothelial cells promote migration and proliferation of enteric neural crest cells via beta1 integrin signaling. Dev Biol 330:263-272, 2009

25. Wallace AS, Barlow AJ, Navaratne L, et al: Inhibition of cell death results in hyperganglionosis: Implications for enteric nervous system development. Neurogastroenterol Motil 21:768-e749, 2009

26. Hao MM, Anderson RB, Kobayashi K, et al: The migratory behavior of immature enteric neurons. Dev Neurobiol 69:22-35, 2009

27. Jacobs-Cohen RJ, Payette RF, Gershon MD, et al: Inability of neural crest cells to colonize the presumptive aganglionic bowel of 1s/1s mutant mice: Requirement for a permissive microenvironment. J Comp Neurol 255:425-438, 1987

28. Wu JJ, Chen JX, Rothman TP, et al: Inhibition of in vitro enteric neuronal development by endothelin-3: Mediation by endothelin B receptors. Development 126:1161-1173, 1999

29. Parikh DH, Tam PK, Van Velzen D, et al: Abnormalities in the distribution of laminin and collagen type IV in Hirschsprung's disease. Gastroenterology 102:1236-1241, 1992

30. Druckenbrod NR, Epstein ML: Age-dependent changes in the gut environment restrict the invasion of the hindgut by enteric neural progenitors. Development 136:3195-3203, 2009

31. Hotta R, Anderson RB, Kobayashi K, et al: Effects of tissue age, presence of neurones and endothelin- 3 on the ability of enteric neurone precursors to colonize recipient gut: Implications for cell-based therapies. Neurogastroenterol Motil 22:331, 2010

32. Burns AJ, Roberts RR, Bornstein JC, et al: Development of the enteric nervous system and its role in intestinal motility during fetal and early postnatal stages. Semin Pediatr Surg 18:196-205, 2009

33. Tam PK, Garcia-Barcelo M: Genetic basis of Hirschsprung's disease. Pediatr Surg Int 25:543-58, 2009

34. Borrego S, Wright FA, Fernandez RM, et al: A founding locus within the RET proto-oncogene may account for a large proportion of appar- 
ently sporadic Hirschsprung disease and a subset of cases of sporadic medullary thyroid carcinoma. Am J Hum Genet 72:88-100, 2003

35. Sanchez-Mejias A, Fernandez RM, Lopez-Alonso M, et al: New roles of EDNRB and EDN3 in the pathogenesis of Hirschsprung disease. Genet Med 12:39-43, 2010

36. Arnold S, Pelet A, Amiel J, et al: Interaction between a chromosome 10 RET enhancer and chromosome 21 in the Down syndrome-Hirschsprung disease association. Hum Mutat 30:771-775, 2009

37. Vohra BP, Planer W, Armon J, et al: Reduced endothelin converting enzyme-1 and endothelin-3 mRNA in the developing bowel of male mice may increase expressivity and penetrance of Hirschsprung disease-like distal intestinal aganglionosis. Dev Dyn 236:106-117, 2007

38. Almond S, Lindley RM, Kenny SE, et al: Characterisation and transplantation of enteric nervous system progenitor cells. Gut 56:489-496, 2007

39. Bixby S, Kruger GM, Mosher JT, et al: Cell-intrinsic differences between stem cells from different regions of the peripheral nervous system regulate the generation of neural diversity. Neuron 35:643-656, 2002

40. Bondurand N, Natarajan D, Thapar N, et al: Neuron and glia generating progenitors of the mammalian enteric nervous system isolated from foetal and postnatal gut cultures. Development 130:6387-6400, 2003

41. Kruger GM, Mosher JT, Bixby S, et al: Neural crest stem cells persist in the adult gut but undergo changes in self-renewal, neuronal subtype potential, and factor responsiveness. Neuron 35:657-669, 2002

42. Natarajan D, Grigoriou M, Marcos-Gutierrez CV, et al: Multipotential progenitors of the mammalian enteric nervous system capable of colonising aganglionic bowel in organ culture. Development 126:157168,1999

43. Suarez-Rodriguez R, Belkind-Gerson J. Cultured nestin-positive cells from postnatal mouse small bowel differentiate ex vivo into neurons, glia, and smooth muscle. Stem Cells 22:1373-1385, 2004

44. Metzger M, Bareiss PM, Danker T, et al: Expansion and differentiation of neural progenitors derived from the human adult enteric nervous system. Gastroenterology 137:2063-73:e2064, 2009

45. Metzger M, Caldwell C, Barlow AJ, et al: Enteric nervous system stem cells derived from human gut mucosa for the treatment of aganglionic gut disorders. Gastroenterology 136:2214-2225:e22112213, 2009 